\title{
RECENSIONES Y RESEÑAS
}

\section{ACERCA DE LAS ENFERMEDADES DEL CURRICULUM}

\author{
Rosalia Bustos Quintero *
}

Hace unos días cayó en mis manos, no por azar sino gracias a la gentileza del profesor Roberto Munizaga, un artículo publicado por Stephen Abrahamson, doctor en Filosofia, profesor en la Escuela de Medicina de la Universidad de California del Sur en Los Angeles.

Su título llamó poderosamente mi atención: Enfermedades del Curriculum **. No era la primera vez que veía expresada la idea de que el curriculum sufriera enfermedades. Sin ir más lejos, en Dos informes sobre educación leemos lo siguiente: "La materia de estudio del liceo, de las escuelas primarias o aún de la Universidad misma, está condenada a una especie de destino fisico, de inevitable destino biológico, que la hace transformarse después de algunos años, perdiendo su contacto con las realidades de la vida. Les sucede a las materias de estudios lo que a todos los seres vivos: envejecen. Y ésto dentro del liceo, de la Universidad, de cualquier escuela; ella se anquilosa. Hay, pues, como una especie de arteriosclerosis de la materia de estudios, que es inevitable: abstracción, verbalismo, irrealidad, desvitalización, etc.»

La tesis es básicamente la misma: cualquiera sea nuestra definición del currículum, en definitiva, no podemos desconocer que ésta es una realidad viva y dinámica, que cambia y se modifica por la influencia $y$ condicionamiento de la planificación, realización y evaluación de las tareas educativas. $\mathrm{Si}$ aceptamos que se trata de una realidad "viva" podemos comprender que se afirme la existencia de un funcionamiento "normal" del curriculum, que es equilibrio y armonia, y se llame "enfermedades" a las formas de funcionamiento desviadas de lo normal que ocasionan tensiones y conflictos.

Resulta, entonces, altamente sugerente y, aún más, una clara invitación a la meditación contingente, la lectura de las nueve enfermedades que el doctor Abrahamson describe. $\mathrm{Al}$ hacerlo, hemos tenido la certeza de que esta taxonomía surgida del análisis de los currícula de las Escuelas de Medicina norteamericanas, ofrece una pauta inestimable para el diagnóstico de las enfermedades de nuestros

- Profesora Facultad de Educación, Universidad de Chile. 


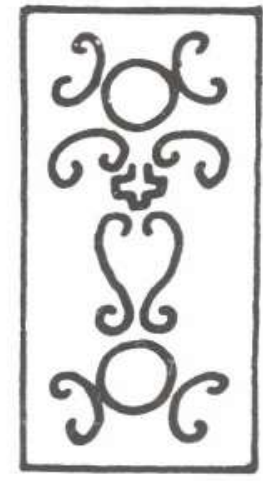

programas de formación de profesores, como de cualesquiera otros. Más allá de la ironía, y de la sonrisa motivada por el uso de términos médicos para referirse a cuestiones educativas, encontramos un pensamiento muy serio y consistente en torno a problemas de organización y funcionamiento del proceso educativo.

Como bien sabemos, un diagnóstico correcto ha de surgir del adecuado análisis de los sintomas. Veamos entonces qué enfermedades curriculares ha logrado identificar el doctor Abrahamson y así sabremos si, en estas descripciones, encontramos elementos que nos permitan un futuro diagnóstico.

1. La primera enfermedad descrita y al mismo tiempo la más frecuente y terrible, es la curriculosclerosis. Ella consiste en un "endurecimiento de las categorias». Toda institución educativa tiene, como parte de su organización administrativa, una serie de servicios o departamentos que constituyen su estructura. Pero cuando los servicios o departamentos tienden a establecer una suerte de territorialidad, oámbito de competencia y dominio, y a imponerse los unos sobre los otros, se convierten en fuerzas inhibidoras del funcionamiento y desarrollo normales del curriculum. En palabras de Abrahamson, en el grado más extremo de curriculosclerosis, *el número de horas asignadas para la enseñanza a un departamento es visto como precio, o al menos la medida, de su importancia. El diseño del currículum, entonces, parece ser más una lucha por el poder que una aventura de planificación educativa*.
Cuando en nuestra experiencia como docentes nos encontramos con instituciones educativas que, estableciendo feudos propios, enfrentan el diseño del currículum como una pugna por obtener más horas para sus programas, deberemos reconocer los sintomas de una curriculosclerosis más o menos acentuada.

2. Otra de las enfermedades que Abrahamson describe es el carcinoma del curriculum. Indetectable en sus primeras etapas, esta enfermedad se caracteriza por un crecimiento, al parecer incontrolable, de un segmento o componente del curriculum. En sus primeras fases, la enfermedad suele confundirse con el ciesarrollo o crecimiento normales que acompañan al avance del conocimiento en una determinada área o al del aumento de las funciones que se asignan a un departamento específico. Este necesario aumento va, generalmente, acompanado de mayores recursos para satisfacer esas mayores necesidades. Pero cuando la asignación de mayores recursos se convierte en un nuevo y mayor crecimiento de las necesidades, se origina un círculo vicioso, un proceso desorbitado y automotivado, en desmedro del desarrollo y crecimiento de los otros sectores del curriculum haciendo que éste se desajuste y desequilibre. Así, pues, planes de estudio e instituciones con un evidente desequilibrio en áreas que los constituyen, muestran sintomas de carcinoma.

3. Otra terrible enfermedad es la curriculoarthritis. Enfermedad sumamente interesante que afecta las articulaciones entre segmentos del curriculum ya sea en sentidovertical u horizontal. Es, básicamente, una dolencia que obstruye los canales de comunicación entre segmentos curriculares. Asi, por ejemplo, puede suceder que los profesores que enseñan las asignaturas de segundo año, desconozcan lo que están haciendo sus colegas de primero: curriculoarthritis vertical. $O$ puede suceder también que dos colegas que enseñan la misma materia en un mismo nivel desconozcan lo que hace cada uno: curriculoarthritis horizontal.

En sus formas benignas, la enfermedad se manifiesta como una especie de pudor entre colegas que, para no ser catalogados de 
"entrometidos", evitan preguntar, consultar, ponerse de acuerdo con quienes trabajan en otro nivel o en su mismo nivel. Se empiezan a producir entonces, repeticiones, vacios e incluso inconsistencias entre los diversos niveles de un programa específico. En sus grados más avanzados, la cirriculoarthritis se manifiesta como hostilidad o resistencia a permitir que colegas de otros campos, se enteren de lo que cada cual está haciendo. En casos de extrema virulencia, la actitud podria resumirse en expresiones del tipo: «No tengo inconvenientes en explicar lo que estoy haciendo, pero ustedes no lo entenderian*.

Nos asiste el convencimiento de que esta dolencia tiene un alto poder de contagio y puede difundirse muy fácilmente. $\mathrm{Y}$ lo peor es que, desgraciadamente, la curriculoarthritis no afecta solamente a las comunicaciones entre los encargados de una asignatura. Afecta también las comunicaciones entre los niveles directivos y operativos, alcanzando en estas esferas grados de increible virulencia.

4. La cuarta enfermedad que Abrahamson describe es la curriculum disesthesia. Se trata de una molestia generalizada, de un estado en que, pareciendo el currículum gozar de buena salud, persiste, sin embargo, la sensación de que algo no está bien. Es posible detectar esta enfermedad muy tempranamente en las opiniones de catedráticos y alumnos que dejan conocer su insatisfacción e inquietud por la forma en que marchan las cosas. No se trata, sin embargo, de las críticas de los reternos disconformesn. En la curriculum disesthesia existe un efectivo y real estado de malestar, en el cual se tiene incluso la evidencia y no sólo la sensación de que las cosas no marchan bien. Lo que sucede es que, en esta enfermedad, uno no puede puntualizar lo que está mal, aun cuando le asiste el convencimiento de que la cosa no marcha. Al definir mejor la naturaleza y extensión de la curriculum disesthesia se logran identificar otras enfermedades curriculares más específicas. Y de ahí que ella aparezca como la fiebre que acompaña a dolencias más graves. Es una enfermedad ssintoma».

5. La enfermedad que se describe a continuación es la curriculitis iatrogénica. Enfer- medad que se caracteriza por lo que podemos llamar *modificaciones permanentes del plan de estudios». En sus estados más agudos, en palabras de Abrahamson, alos constantes acomodos, cambios, modificaciones y ajustes no permiten la revisión reflexiva, y hacen abandonar la investigación evaluadora. Es como si la institución, siendo incapaz de hacer un diagnóstico de lo que está malo en el curriculum, respondiera a esta incapacidad con intervenciones múltiples de una manera muy parecida a la de "ametralladora" atribuida a algunos médicos que tienden a recetar más -y quieren intervenir más- cuando no están seguros del diagnóstico.

En lo que se refiere a esta enfermedad es preciso hacer una aclaración: no se trata de que el currículum no deba ajustarse, modificarse o cambiar en respuesta a fuerzas tales como necesidades de estudiantes y catedráticos, avance de las ciencias, etc. Un currículum que no cambia, que permanece inmutable ante nuevas situaciones, es un currículum en peligro. La mejor manera de hacer que un curriculum entre en crisis es inmovilizarlo. Tales necesarias modificaciones dificilmente podrian considerarse curriculitis iatrogénica. La enfermedad consiste en que se procede a efectuar cambios a tontas y a locas, irreflexivamente, por el prurito de cambiar y sin evaluación previa. Cuando esto sucede se produce la curriculitis iatrogénica que afecta a los programas y planes de estudios haciéndolos perder su estilo y sentido.

\section{Muy similar a la} disesthesia curricular es la enfermedad que Abrahamson llama curriculitis idiopática. Se trata, también en este caso, de un malestargeneralizado, de un general sentimiento de que algo anda mal, pero en la curriculitis idiopática los malestares parecen centrarse explicitamente en la docen-

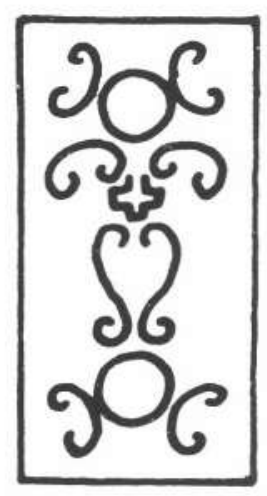


cia. Dice Abrahamson, *aunque la molestia, la indisposición, la insatisfacción se expresa abiertamente y el currículum aparece como el loco... un examen más detenido revela que es la enseñanza la que está mal, no el currículum". Dicho de otra manera, en algunos casos, no es el plan de estudios lo malo, sino lo que los docentes (y administradores, agregamos) hacen con él. Como un ejemplo de esta enfermedad menciona el comentario de un alumno de Medici-
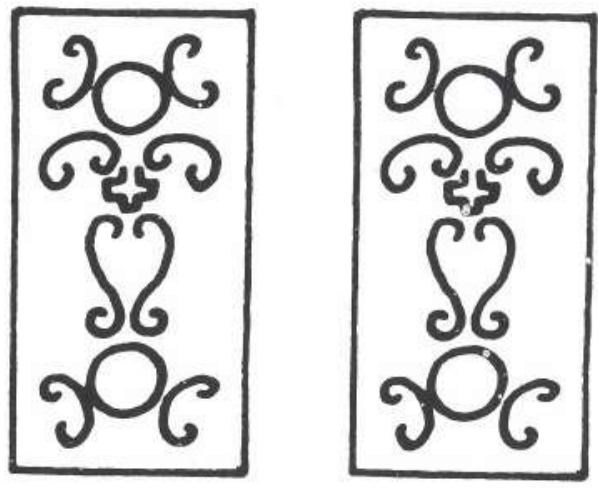

na en unas jornadas de evaluación de un "nuevo plan de estudios" en que defensores y críticos del "viejo plan" y del "nuevo plan" no podian entenderse: «Ustedes saben de qué estamos hablando nosotros los estudiantes. Lo que a nosotros nos preocupa es la diferencia entre la buena enseñanza y la mala enseñanza, no entre el viejo y el nuevo currículum".

En definitiva, esta enfermedad tiene como microbìos generadores a los docentes. Es una enfermedad producida por los malos profesores. Aquel comentarịo de ese estudiante de Medicina lo pone en evidencia. Diriamos que ningún plan de estudios es bueno en manos de docentes mediocres, faltos de originalidad e iniciativa y que un pésimo plan de estudios puede ser muy formativo en manos de profesores que han hecho del enseñar y del aprender una tarea vital y creadora.

7. Otra de las enfermedades descritas por el autor, la hipertrofia del curriculum o curriculomegalia, es -a nuestro juicio- una enfermedad extendida a lo largo de los diversos niveles del sistema escolar. Consiste en que, como consecuencia de la llamada «explosión del conocimiento" se incluye en cada programa de asignaturas una cuota cada vez más amplia de saber, estimada imprescindible para el ejercicio de las funciones de que se trate. Esta inclusión de conocimientos, realizada $\sin$ disminuir lo que ya incluye la asignatura, "conduce necesariamente a un curriculum en creciente atiborramiento en la medida en que más y más conocimientos

apretujan, en lapsos limitadosn.

Los primeros signos de la enfermedad se encuentran en ei incremento de las horas destinadas a transmitir información en desmedro de las sesiones prácticas, y en lo que nosotros hemos traducido como "complejo de cobertura" o "complejo de pasar materia*.

Dos ejemplos señalados por Abrahamson resisltan especialmente sugerentes. Uno de ellos es el comentario que recogiera de alumnos de una escuela de Medicina norteamericana: ‘Tenemos el mejor curso de anatomía del país; lo que los profesores no cubren en las clases o en los laboratorios, lo cubren en el examen finalw. El otro se refiere a un curso que era la permanente víctima de la hipertrofia del curriculum que sufrian otros. Es característica de esta enfermedad que los docentes de las asignaturas que padecen de curriculomegalia tengan una declarada aversión a quitar contenidos de sus propias asignaturas (aún obsoletos) y no tengan el menor escrúpulo en reducir a otras. Así, en el caso de la asignatura mencionada, cada vez que otra disciplina requeria de más tiempo, ella sufria las consecuencias porque se consideraba que "de todas maneras, tenía asignado demasiado tiempon.

8. La curriculosis intercurrente y la osificación curricular son las dos últimas enfermedades mencionadas por Abrahamson. La primera de ellas se caracteriza porque puede presentarse junto con cualquiera de las otras y consiste en que el currículum se desentiende del contexto social, de los problemas y cuestiones propias de su época histórica. Como decía Munizaga en la cita inicial, pierde contacto con las realidades de la vida, se desvitaliza. Esta 
enfermedad no se presenta siempre; puede aparecer o no según las condiciones y circunstancias de la situación. Su curso tampoco es claro, y, en la medida en que suele presentarse con otras enfermedades, suele no ser detectada y, por lo mismo, no tratada. Sus sintomas más evidentes se encuentran en una *suerte de rebeldía de los estudiantes, en directa proporción con el grado de «separación" de la escuela de la sociedad y con el grado de sactivismo" y/o conciencia social entre los estudiantes.

Abrahamson menciona un ejemplo de curriculitis intercurrente tomado de la historia de la educación en los tiempos paleolíticos, y que aparece en la obra de J. Abuer Palliwell: el llamado «Currículum del Tigre Colmillos de Sable».

Se trata de que, en esos tiempos, se generó un currículum que incluía el enseñar a los niños cómo ahuyentar al Tigre Colmillos de Sable con el uso del fuego. Con el correr del tiempo, ese animal se extinguió completamentey, sin embargo, en el curriculum de los niños prehistóricos persistiô -sin alteraciones- la actividad de *amedrentamiento del Tigre Colmillo de Sable por medio del fuegon. En nuestra época, afirma Abrahamson, "la curriculitis intercurrente puede encontrarse en las escuelas de Medicina que insisten en que su verdadera misión es la preparación de cientificos, licenciados e investigadores -y no de médicos- a pesar de la reconocida y aceptada función de preparar a los estudiantes para el ejercicio de la medicinan.

9. Finalmente tenemos la ya mencionada osificación curricular. Enfermedad epidémica que, al parecer, afecta, en una considerable medida, a todos los currícula. Consiste en un endurecimiento que los hace rigidos y resistentes al cambio. Se anquilosan. Esta enfermedad se parece a la curriculoarthritis descrita anteriormente. Pero, en tanto que aquella afectaba a segmentos curriculares obstruyendo las comunicaciones, la osificación curricular afecta a todos los componentes del currículum haciéndolos parecer "modelados en concreto". Los currícula afectados por esta dolencia tienen la rigidez de un cadáver y cualquier intento por innovarlos tropieza con insalvables dificultades.

La lista de enfermedades presentadas no es más que una lista inicial. Ella reune una extensa gama de dolencias que van desde aquellas que se caracterizan por una excesiva intervención hasta las que reflejan indiferencia y, finalmente, «rigor mortis». El propósito del autor ha sido contribuir a la temprana detección de sus sintomas para tratarlas y sobrevivir a ellas. Sus palabras finales suenan como advertencia y programa de acción: «Ojalá podamos aprender a detectar tempranos signos de estas enfermedades; ojalá podamos estudiar y desarrollar su etiología. Así como el hombre -en sus primeros tiempos-, vivió entre enfermedades sin conocerlas ni a ellas ni a su etiologia, nosotros no solo hemos vivido en medio de las enfermedades del curriculum sino que hemos sobrevivido a ellas, y todavia más, en algunos casos, las hemos tratado correctamente, sea por accidente o, en el mejor de los casos, por intuición. Pero si, conceptualmente, el curriculum es un ser vivo (o al menos, dinámico) aquellos que son responsables de su administración tienen la obligación de procurar el mejor desarrollo mediante un manejo inteligente e informado, quizás a través de la comprensión de los procesos patológicos".

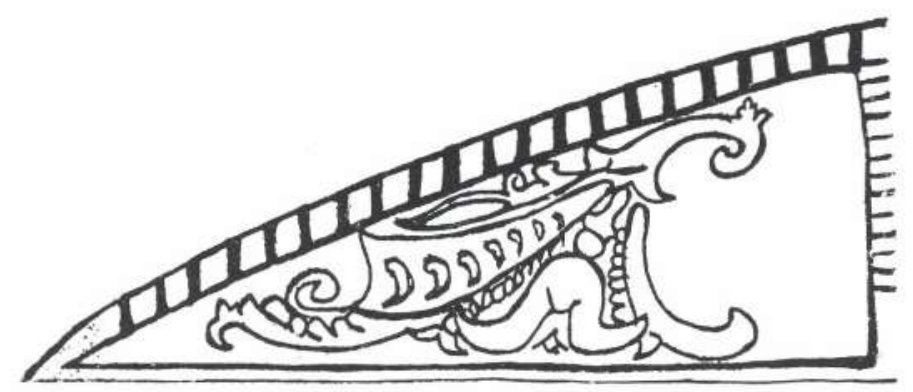

